\title{
Magnetization dynamics in the presence of pure spin currents in magnetic single and double layers in spin ballistic and diffusive regimes
}

\author{
O. Mosendz, ${ }^{1}$ G. Woltersdorf, ${ }^{2}$ B. Kardasz,${ }^{3}$ B. Heinrich, ${ }^{3}$ and C. H. Back ${ }^{2}$ \\ ${ }^{1}$ Materials Science Division, Argonne National Laboratory, 9700 S. Cass Avenue, Argonne, Illinois 60439, USA \\ ${ }^{2}$ Universität Regensburg, Universitätsstraße 31, 93040 Regensburg, Germany \\ ${ }^{3}$ Simon Fraser University, 8888 University Drive, Burnaby, British Columbia, Canada V5A 1S6
}

(Received 23 March 2009; revised manuscript received 21 May 2009; published 12 June 2009)

\begin{abstract}
In this paper we study the spin transport by using the spin-pumping effect in epitaxial magnetic single and double layer film structures. For the magnetic single layer sample we show the spin-pumping-induced interface damping increases and saturates with the Au capping layer thickness. In addition magnetic double layer structures allowed us to investigate both the spin-pump and spin-sink effects. Coupling of pure spin currents to the magnetization via spin-sink effect is studied using time-resolved magneto-optical Kerr effect. These measurements were used to study the propagation of pure spin currents across a Au spacer layer between the two ferromagnets. The propagation of spin momentum density through the Au spacer layer was well described by spin-diffusion equation, which takes into account electron momentum and spin-flip scattering. The spindiffusion theory was integrated into modified Landau-Lifshitz equations accounting in self-consistent manner for spin-pump/sink mechanism and spin momentum density propagation. Good agreement between theory and experimental data was found.
\end{abstract}

DOI: $10.1103 /$ PhysRevB.79.224412

PACS number(s): 75.70.Cn, 75.70.Ak

\section{INTRODUCTION}

Information in complementary metal-oxide semiconductor and data storage technologies is mainly transported and manipulated by charge currents. With accelerating miniaturization of semiconducting devices, heat dissipation and power consumption become significant obstacles to further technological advances. Therefore researchers in academia and industry are searching for alternative technologies, which could circumvent these problems. One promising candidate to replace the existing charge-based technology is based on spin currents rather than charge currents; this effort is usually coined by spintronics. Hybrid devices employing spin-polarized charge currents are already actively implemented as hard drive read heads and nonvolatile magnetic random access memory. They proved to be efficient and contributed to further device miniaturization. In our work we concentrated on studies of the pure spin currents, which are not accompanied by the charge current. Using pure spin currents for information transfer and processing requires understanding of the following processes: (a) generation of pure spin currents; (b) propagation of pure spin currents in normal metals; and (c) coupling of pure spin currents to magnetization. In this paper the above questions are studied on single and double layer magnetic ultrathin films.

Spintronic devices are based on ultrathin magnetic films. In order to control the magnetization dynamics one needs to understand and control the relaxation processes in these systems. Spin dynamics in the limit of ultrathin ferromagnetic films can be described by the Landau-Lifshitz-Gilbert (LLG) equation of motion,

$$
\frac{1}{\gamma} \frac{\partial \vec{M}}{\partial t}=-\left[\vec{M} \times \vec{H}_{\mathrm{eff}}\right]+\frac{\alpha}{\gamma}\left[\vec{M} \times \frac{\partial \vec{n}}{\partial t}\right],
$$

where $\vec{n}$ is the unit vector in the direction of the magnetization $\vec{M}, \gamma=g e / 2 m c$ is the absolute value of the gyromagnetic ratio, and $\alpha$ is the dimensionless Gilbert damping parameter. The first term on the right-hand side represents the precessional torque in the internal field $\vec{H}_{\text {eff }}$ and the second term represents the Gilbert damping torque. ${ }^{1,2}$

The ferromagnetic resonance (FMR) is a convenient tool to determine the Gilbert damping parameter $\alpha$. For this purpose one measures the FMR linewidth $[\Delta H$, half width at half maximum] of the imaginary part of the rf susceptibility $\chi^{\prime \prime}$ which is usually measured at a constant microwave frequency by sweeping the dc-applied field. For Gilbert damping $\Delta H$ is strictly linearly dependent on the microwave angular frequency $\omega, \Delta H=\alpha \frac{\omega}{\gamma}$. Gilbert damping in metallic ferromagnets is caused by incoherent scattering of electronhole pairs which are excited by resonant spin wave (see review of relaxation processes in Ref. 2).

In magnetic single and multilayers separated and covered by nonmagnetic spacers one needs to include an additional source of Gilbert damping. It arises from dynamic nonlocal spin transport. Tserkovnyak et al. $^{3}$ and Heinrich et $a l^{4}$ showed that an interface damping can be generated by pumping spin current from a ferromagnet $(\mathrm{F})$ into adjacent layers (see Fig. 1). The spin current is generated by a precessing magnetic moment in the layer F1 at the F1/normal-metal (NM) interface.

The precessing magnetization at the F1/NM interface acts as a "peristaltic spin pump" which creates an accumulated dynamic magnetic-moment density in NM. The spin current perpendicular to the F1/NM interface is given by

$$
\vec{I}_{\mathrm{sp}}=\frac{\hbar}{8 \pi} \operatorname{Re}\left(2 g_{\uparrow \downarrow}\right)\left[\vec{n} \times \frac{\partial \vec{n}}{\partial t}\right],
$$

where $g_{\uparrow \downarrow}$ is the spin mixing conductance which is determined by the transmission $\left(t_{l}^{\uparrow}, t_{l}^{\downarrow}\right)$ and reflection coefficients $\left(r_{l}^{\uparrow}, r_{l}^{\downarrow}\right)$ for the majority and minority spins in NM, respectively. For FM layers thicker than the spin coherence length 


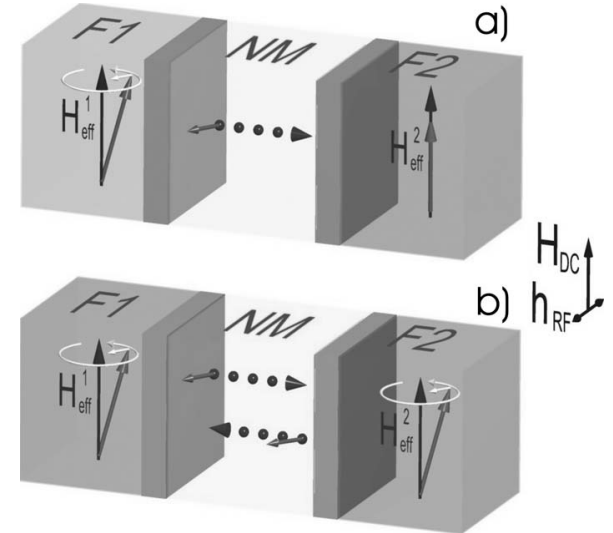

FIG. 1. Schematic model of spin-pump/spin-sink mechanism in magnetic double layers.

(a few atomic layers) the spin mixing conductance is given by

$$
g_{\uparrow \downarrow}=\sum_{l}\left[1-\operatorname{Re}\left(r_{l}^{\uparrow} r_{l}^{\downarrow^{*}}\right)\right] \approx 0.75 n^{2 / 3}
$$

where $l$ is the number of transverse spin channels, $g_{\uparrow \downarrow}$ is in units of $e^{2} / h$, and $n$ is the density of electrons per spin in NM. NM metal spacer acts as a node in magnetoelectrics circuitry due to diffuse scattering of NM electrons at the FM/NM interfaces. ${ }^{5}$ This diffuse scattering leads to a random distribution of electrons in the $k$ space which allows one to use different chemical potentials for the majority and minority electrons consequently allowing one to introduce the accumulated spin density $\overrightarrow{\mathbf{m}}_{\mathrm{NM}}$,

$$
\overrightarrow{\mathbf{m}}_{\mathrm{NM}}=\frac{\hbar}{2} N\left(\varepsilon_{F}\right) \vec{\mu}_{s},
$$

where $\hbar$ is a Planck constant, $N\left(\varepsilon_{F}\right)$ is the Fermi-level density of states (per spin and unit volume) in the NM node, and $\vec{\mu}_{s}$ is the difference between the chemical potentials for spin up and down electrons. The accumulated spin density $\overrightarrow{\mathbf{m}}_{\mathrm{NM}}$ is a quantity close to experimental observations and will be further used in this paper instead of $\vec{\mu}_{s}$ which is commonly used in theoretical articles. The spin current can propagate in normal metals in ballistic limit if the NM spacer thickness is much larger than the electron-spin mean-free path. In the ballistic limit spin density in NM $\overrightarrow{\mathbf{m}}_{\mathrm{NM}}$ propagates with the Fermi velocity $v_{F}$ of itinerant electrons with equal probability in both directions normal to the interface. This means that half of the spin density in NM will propagate away from the interface, carrying magnetic moment away from the pumping ferromagnet, while the other half will lead to a spin backflow. The spin backflow partly compensates spin pumping and is an important part of magnetoelectronic circuitry. It is described in Ref. 5 by the mixing conductance term in Eq. (26). Note that this term is perpendicular to the instantaneous magnetization, so it is fully absorbed at the FM/NM interface. The total spin-current balance is given by

$$
\vec{I}_{\mathrm{sp}}-0.5 v_{F} \overrightarrow{\mathbf{m}}_{\mathrm{NM}}=0.5 v_{F} \overrightarrow{\mathbf{m}}_{\mathrm{NM}},
$$

where the left side of this equation describes the net flow of the spin current across the F1/NM interface. In this expression one assumes that the returning spin current is entirely absorbed at the F1/NM interface and the forward spin current is absorbed by another magnetic layer F2 (at the NM/F2 interface) with the magnetic moment parallel to F1. This is strictly correct only for small precessional angle where the pumped spin momentum is nearly perpendicular to the saturation magnetization moment ${ }^{6,7}$ and the $\mathrm{F} 1 / \mathrm{NM}$ and NM/F2 interfaces act as ideal spin sinks. Note that only half of the pumped spin current $\vec{I}_{\mathrm{sp}}$ is directed to F2.

In magnetic double layers $\mathrm{F} 1 / \mathrm{NM} / \mathrm{F} 2$, with parallel static magnetic moments, the second NM/F2 interface acts as an ideal spin sink. The pumped magnetic momentum from the F1/NM interface is fully absorbed at the NM/F2 interface providing an effective spin brake for the precessing magnetic moment in F1 (i.e., loss of spin angular momentum). The conservation of total angular momentum leads to an additional damping in the F1 layer, which follows the phenomenology of Gilbert damping; i.e., the damping effective field is proportional to $\vec{M} \times \partial \vec{M} / \partial t$. In addition the strength scales inversely with the ferromagnetic film thickness ${ }^{8,9}$ as a consequence of the interface nature of spin pumping,

$$
\frac{G_{\mathrm{sp}}}{\gamma}=\frac{g \mu_{B}}{4 \pi} g_{\uparrow \downarrow} \frac{1}{d},
$$

where $\mu_{B}$ is the Bohr magneton and $d$ is the thickness of the F1 layer. Two processes occur at the interfaces: spin current is generated (spin pump) by precessing magnetization and the spin current (originating from the other ferromagnetic layer) is absorbed (spin sink). Evidence for this effect was found in the FMR line broadening observed in magnetic single $^{10}$ and double layers ${ }^{4,8}$ (see further details in review article). ${ }^{7}$ In magnetic single layers at large precessional amplitudes spin pumping induces a dc spin momentum accumulation which generates a measurable dc voltage across the FM/NM interface. ${ }^{11}$

The spin-pump/spin-sink effect can be accounted for in the LLG equation of motion by adding two terms. For small precessional angles the magnetization dynamics in the presence of ballistic dynamic exchange coupling can be described by the following set of coupled Landau-LifshitzGilbert equations: ${ }^{4}$

$$
\begin{aligned}
\frac{d \vec{n}_{i}}{d t}= & -\mu_{0} \gamma\left[\vec{n}_{i} \times \mathbf{H}_{\mathrm{eff}}\right]+\alpha_{i}\left[\vec{n}_{i} \times \frac{d \vec{n}_{i}}{d t}\right] \\
& +\alpha_{i}^{\mathrm{sp}}\left[\vec{n}_{i} \times \frac{d \vec{n}_{i}}{d t}-\vec{n}_{j} \times \frac{d \vec{n}_{j}}{d t}\right],
\end{aligned}
$$

where $\vec{n}_{i}$ are the unit vectors along the instantaneous magnetization directions in $\mathrm{F} 1$ and $\mathrm{F} 2, i, j=1,2$ and $j \neq i$. The strength of the spin-pump and spin-sink effects is given by the parameter $\alpha_{i}^{\mathrm{sp}}=g \mu_{B} \frac{g_{\uparrow \downarrow}}{\mu_{0} M_{\mathrm{s}}} \frac{1}{d_{i}}$, where $d_{i}$ is the corresponding film thickness. ${ }^{4,12}$ The exchange of spin currents is a symmetric concept and the equation of motion for the other ferromagnetic layer is obtained by interchanging the indices 
$i \rightleftarrows j$ in Eq. (7). The third and fourth terms on the right-hand side of Eq. (7) represent the spin-pump and spin-sink effects, respectively. The third term is generated by the spin pumping of the layer $i$ and the fourth term in Eq. (7) corresponds to the absorbed spin current in the layer $i$ due to spin pumping from the layer $j$. The signs (+) and (-) in the third and fourth terms in Eq. (7) represent the spin-current directions. When the resonance frequencies are equal in both ferromagnetic films, then the net transfer of spin momentum across the F1/NM and NM/F2 interfaces can be compensated resulting in zero interface damping. ${ }^{4}$

The mutual exchange of spin currents between F1 and F2 leads to dynamic exchange coupling. ${ }^{4,13}$ In contrast to static interlayer exchange coupling this coupling does not oscillate with the spacer thickness, it is almost independent of interface roughness, and its range is limited only by the spindiffusion length. ${ }^{4}$ At present evidence of this coupling is based on two types of experiments: (a) broadening of the FMR lines ${ }^{8}$ (see additional references in the review article $^{7}$ ) and (b) spin-current-induced dynamics. ${ }^{14,15}$ In the following we will show the results from measurements in magnetic single and double layers using both methods.

\section{DIFFUSIVE SPIN MOMENTUM TRANSFER}

The accumulated spin density moves across a thick NM spacer by spin-diffusion process. The motion of itinerant electrons in NM is affected by momentum (leading to resistance) and spin-flip scattering (leading to loss of spin momentum). The spin-flip scattering in NM is caused by spinorbit coupling. This means only some momentum scattering events also lead to a spin flip. ${ }^{16}$ The spin-diffusion length $\delta_{\text {sd }}$ determines the length scale of the spin momentum decay. For spacers significantly thinner than $\delta_{\text {sd }}$ the loss of spin momentum is negligible and the spin-current propagation can be considered to be in a nearly spin ballistic regime.

At the present time there is no nonlocal spin transport theory allowing one to cover the spin transport in NM spacers from the ballistic to spin-diffusion regime. In order to cover thick NM spacers we use the spin-diffusion equations. In this case the time dependence of the accumulated magnetic-momentum density $\vec{m}_{\mathrm{NM}}$ in NM is described by the diffusion equation,

$$
i \omega \vec{m}_{\mathrm{NM}}=D \frac{\partial^{2} \vec{m}_{\mathrm{NM}}}{\partial x^{2}}-\frac{1}{\tau_{\mathrm{sf}}} \vec{m}_{\mathrm{NM}}
$$

where $\omega$ is the angular frequency, $\tau_{\text {sf }}$ is the spin-flip time, $x$ is the coordinate normal to the interface, and $D=v_{F}^{2} \tau_{\mathrm{el}} / 3$ is the diffusion coefficient. $\tau_{\mathrm{el}}$ is the electron momentum relaxation time. ${ }^{17}$ Equation (8) determines the spin-diffusion length $\delta_{\text {sd }}$ in NM. For $1 / \tau_{\mathrm{sf}} \gg \omega$ the spin-diffusion length, $\delta_{\mathrm{sd}}$, is given by

$$
\delta_{\text {sd }}=\left(D \tau_{\text {sf }}\right)^{0.5}
$$

The solution of Eq. (8) requires boundary conditions. For a single magnetic layer structure F/NM the boundary condition at the F/NM interface is given by ${ }^{17}$

$$
\vec{I}_{\mathrm{sp}}-\frac{1}{2} v_{F} \vec{m}_{\mathrm{NM}}=-D \frac{\partial \vec{m}_{\mathrm{NM}}}{\partial x}
$$

While for the outer interface we use the free-magneticmoment condition

$$
\frac{\partial \vec{m}_{\mathrm{NM}}}{\partial x}=0 .
$$

For a magnetic double layer structure F1/NM/F2 the boundary condition at the $\mathrm{F} 1 / \mathrm{NM}$ interface is equivalent to $\mathrm{Eq}$. (10). The boundary condition at the NM/F2 interface is ${ }^{17}$

$$
-\frac{\partial \vec{m}_{\mathrm{NM}}}{\partial x}=\frac{1}{2} v_{F} \vec{m}_{\mathrm{NM}} \text {. }
$$

The boundary conditions in Eqs. (11) and (12) are valid for the case when the layer F2 is off resonance and therefore contributes very little to spin pumping. The coefficient 0.5 corresponds to the effective transmission coefficient from the NM to F layers and is given by Eq. (13) in Ref. 17. The right-hand side of Eq. (12) represents the spin current from $\mathrm{NM}$ into F2 and acts as a driving torque for the magnetic moment in F2.

The main purpose of this paper is to extend the understanding of the spin-pump/sink effects in magnetic multilayers with the NM spacer operating from spin ballistic to diffuse limits. To this purpose we investigated the following trilayer structures: $20 \mathrm{Au} / 12 \mathrm{Fe} / 300 \mathrm{Ag} / 16 \mathrm{Fe} / \mathrm{GaAs}$ and $20 \mathrm{Au} / 12 \mathrm{Fe} / n \mathrm{Au} / 16 \mathrm{Fe} / \mathrm{GaAs}$ with $n=150,200,250$, and 300 , where integers represent number of monolayers (MLs). Measurements on this set of samples were complemented with a series of single magnetic layer samples covered by a NM layer: $n \mathrm{Au} / 16 \mathrm{Fe} / \mathrm{GaAs}$, where $n=20,80,150,200,250$, and 300 .

\section{SAMPLE PREPARATION}

The metallic thin films for our experiments were grown by molecular beam epitaxy (MBE) on GaAs(001) singlecrystal templates. $\mathrm{GaAs}(001)$ wafer was used as a substrate for several reasons. First, its lattice constant is only $1.4 \%$ smaller than the double of the lattice constant of bcc Fe, allowing one to grow high-quality crystalline epitaxial $\mathrm{Fe}(001)$ ultrathin films. Second, the Fe/GaAs(001) interface creates a strong in-plane uniaxial magnetic interface anisotropy ${ }^{8}$ which was used to separate the FMR signals in $\mathrm{Fe} / \mathrm{NM} / \mathrm{Fe} / \mathrm{GaAs}(001)$ multilayers. The Fe films were deposited at room temperature on $4 \times 6-\mathrm{GaAs}(001)$ reconstructed surfaces (see Fig. 2). The $4 \times 6$ surface reconstruction was obtained by annealing the GaAs wafer at $\simeq 600{ }^{\circ} \mathrm{C}$ following a hydrogen cleaning and grazing incidence $\mathrm{Ar}^{+}$sputtering at $650 \mathrm{eV}$. The GaAs wafer was rotated around its normal during $\mathrm{Ar}^{+}$sputtering. The $4 \times 6$ reconstruction is a pseudoreconstruction which consists of $2 \times 6$ and $4 \times 2$ regions. It is worthwhile to point out that even though reflection highenergy electron diffraction (RHEED) showed a relatively strong intensity for the $4 \times 2$ reconstruction the in situ scanning tunneling microscopy (STM) revealed that the $4 \times 2$ reconstruction occupies only $5 \%$ of the GaAs(001) surface. 


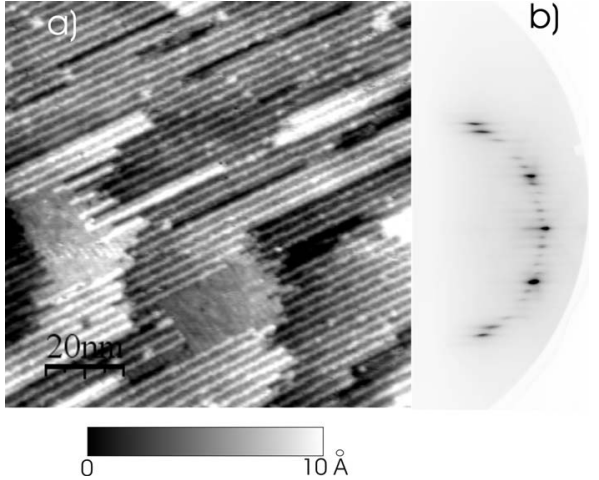

FIG. 2. Pseudo- $4 \times 6$ reconstruction of semi-insulating GaAs template. (a) shows a STM image with the $2 \times 6$ regions with rows of As dimers oriented along the $[1 \overline{1} 0]$ crystallographic direction and the $4 \times 2$ region with rows of $\mathrm{Ga}$ dimers running along the [110] crystallographic direction. (b) RHEED diffraction pattern with the primary electron beam along the $[1 \overline{1} 0]$ direction.

The metal films were deposited at a rate of $2 \mathrm{ML} / \mathrm{min}$. The growth was monitored by RHEED intensity oscillations, which persisted for thicknesses of up to 40 atomic layers for all deposited metallic films (Fig. 3).

Continuous films are formed starting from the third atomic layer. At this thickness the RHEED specular spot intensity strongly increased [see Fig. 4(a)] and the STM images showed a continuous film. Films up to $40 \mathrm{ML}$ were grown in a quasilayer-by-layer mode with the unfilled atomic layers mostly confined to the top two atomic layers. A highcrystalline quality and smooth interfaces were confirmed also by the RHEED diffraction patterns, plan-view transmission electron microscope (TEM) images, ${ }^{18}$ cross-sectional TEM (cf. Fig. 5), and STM [see Fig. 4(b)]. Thick Au layers (over 200 atomic layers) showed some screw dislocations causing the surface roughness to be extended to about three atomic layers.

\section{MAGNETIC PROPERTIES}

The role of spin currents on the interface Gilbert damping was studied using a $20 \mathrm{Au} / 12 \mathrm{Fe} / 300 \mathrm{Ag} / 16 \mathrm{Fe} / \mathrm{GaAs}(001)$

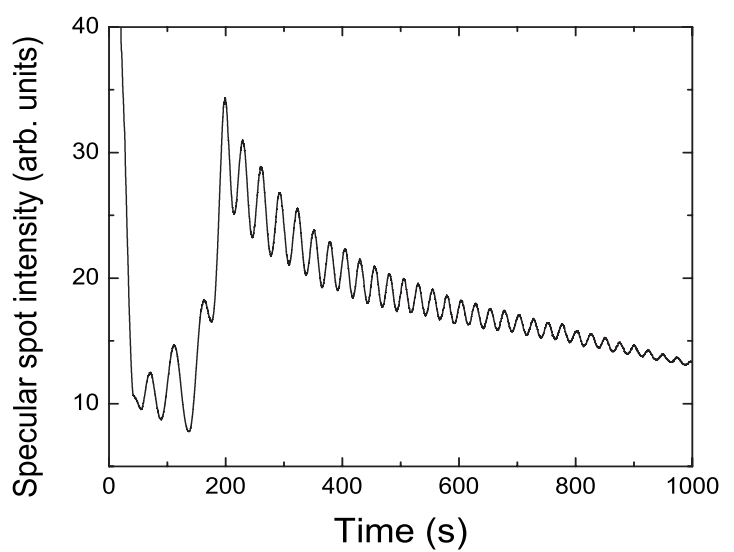

FIG. 3. RHEED specular spot intensity oscillations of 20 atomic layers of Fe deposited on a GaAs(001) template.
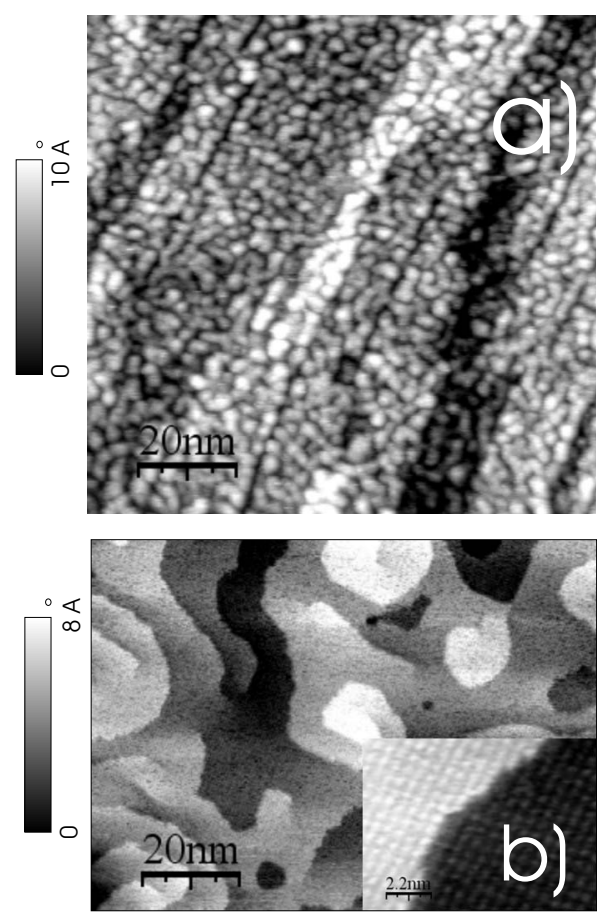

FIG. 4. (a) STM image of 2 ML Fe deposited on GaAs(001). (b) STM of $200 \mathrm{Au} / \mathrm{Fe} / \mathrm{GaAs}(001)$ shows atomic terraces with an average size of $\approx 15 \mathrm{~nm}$. The inset shows a subatomic resolution STM image of a single-crystal $2 \times 2$ reconstructed Au surface

structure, where the integers represent the number of atomic layers.

\section{A. Static magnetic properties}

The magnetic anisotropies for the magnetic trilayer 20Au/ $12 \mathrm{Fe} / 300 \mathrm{Ag} / 16 \mathrm{Fe} / \mathrm{GaAs}(001)$ structure were obtained by standard FMR measurements. ${ }^{19}$ The in-plane angular dependence of FMR at $24 \mathrm{GHz}$ [see Fig. 6(a)] results in the following magnetic parameters. The bottom $16 \mathrm{Fe}$ layer grown on GaAs(001): the in-plane uniaxial anisotropy energy $K_{u}$ $=-4.45 \times 10^{5} \mathrm{erg} / \mathrm{cm}^{3}$ with the hard magnetic axis along the

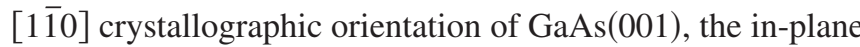
fourfold anisotropy energy $K_{1}=2.65 \times 10^{5} \mathrm{erg} / \mathrm{cm}^{3}$, and the effective demagnetizing field $4 \pi M_{\text {eff }}=15.96 \mathrm{kG}$. The top $12 \mathrm{Fe}$ film grown on the $\mathrm{Ag}(001)$ layer: $K_{1}=3.67$ $\times 10^{5} \mathrm{erg} / \mathrm{cm}^{3}, K_{u}=8.3 \times 10^{3} \mathrm{erg} / \mathrm{cm}^{3}$ with the easy magnetic axis along the $[1 \overline{1} 0]$, and $4 \pi M_{\text {eff }}=14.65 \mathrm{kG}$.

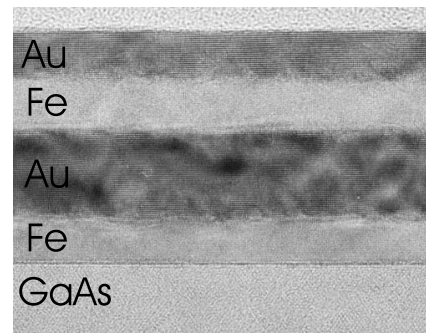

FIG. 5. High-resolution cross-sectional TEM image of a 20Au/ $40 \mathrm{Fe} / 40 \mathrm{Au} / 31 \mathrm{Fe} / \mathrm{GaAs}$ multilayer. 

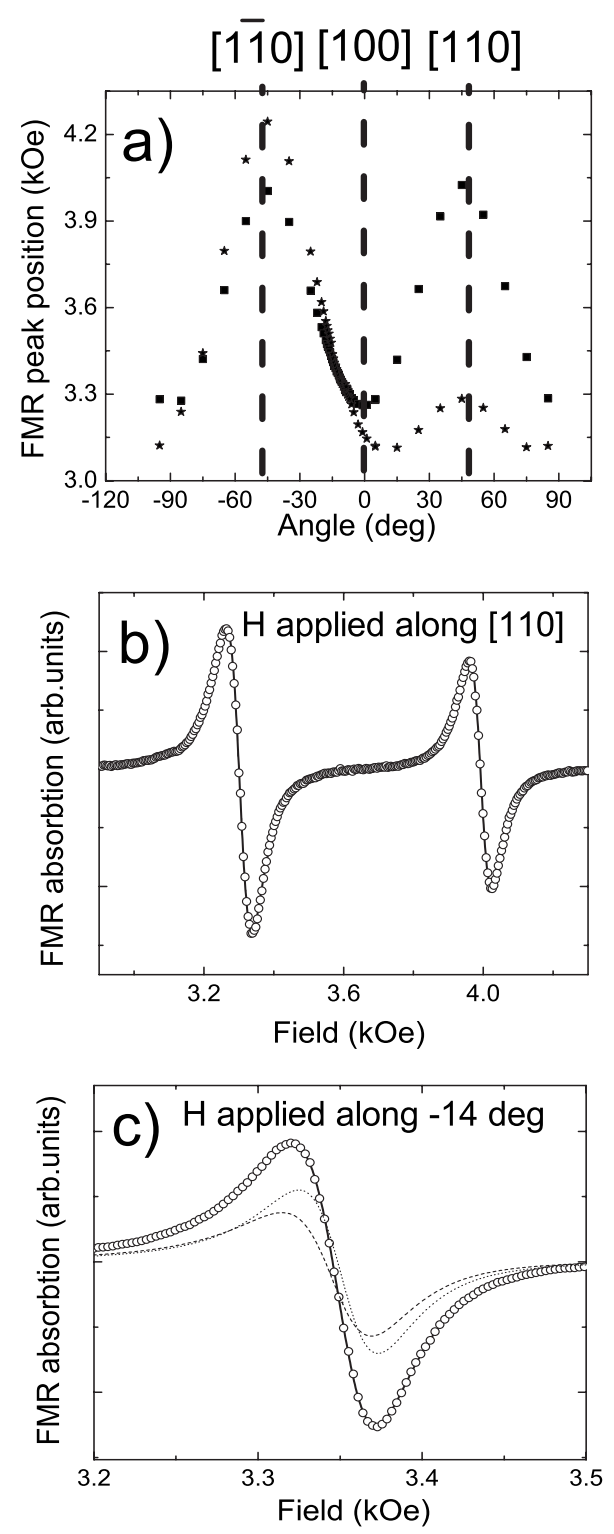

FIG. 6. FMR studies at $24 \mathrm{GHz}$ using $20 \mathrm{Au} / 12 \mathrm{Fe} / 300 \mathrm{Ag} / 16 \mathrm{Fe} /$ $\mathrm{GaAs}(001)$. The sample was inserted inside a microwave cavity which provided parallel and symmetric driving rf fields in the $12 \mathrm{Fe}$ and 16Fe films. (a) The FMR peak positions as a function of the angle of the in-plane magnetic field with respect to the [100] crystallographic axis. The $\square$ and $\star$ points correspond to $12 \mathrm{Fe}$ and $16 \mathrm{Fe}$, respectively. (b) The FMR signal with the external field along the [110] axis. The FMR signals of the two layers are well separated in field. (c) shows the FMR signal at the accidental crossover of the FMR fields. The dashed and the dotted lines were obtained by fitting the data with the field derivatives of two Lorentzian lines. The solid line shows resulting fit.

The $16 \mathrm{Fe}$ layer possesses a large in-plane uniaxial magnetic crystalline anisotropy. The interface uniaxial anisotropy in $\mathrm{Fe} / \mathrm{GaAs}(001)$ layers is inversely proportional to the $\mathrm{Fe}$ layer thickness. ${ }^{8}$ This interface uniaxial anisotropy allowed one to separate the resonance fields of the $16 \mathrm{Fe}$ and $12 \mathrm{Fe}$ films for most angles except a narrow crossover region [see Fig. 6(a)].

\section{B. Dynamic magnetic properties}

Gilbert damping and spin-pumping contributions in 20Au/ $12 \mathrm{Fe} / 300 \mathrm{Ag} / 16 \mathrm{Fe} / \mathrm{GaAs}(001)$ were determined by FMR. The thickness of the $\mathrm{Ag}$ spacer in this structure is $60 \mathrm{~nm}$. The spin-diffusion length in Ag at RT is expected to be $\approx 150 \mathrm{~nm}$ in single crystalline $\mathrm{Ag} .{ }^{15}$ Hence the spin-electron transport is between the ballistic and spin-diffusion limits. The level of spin diffusion for the $60 \mathrm{~nm} \mathrm{Ag} \mathrm{spacer} \mathrm{thickness} \mathrm{was} \mathrm{tested}$ by preparing $20 \mathrm{Au} / 5 \mathrm{Ag} / 16 \mathrm{Fe} / \mathrm{GaAs}(001)$ and $20 \mathrm{Au} / 300 \mathrm{Ag} /$ $16 \mathrm{Fe} / \mathrm{GaAs}(001)$ samples. The magnetic damping parameter $\alpha$ of the $16 \mathrm{Fe}$ film in $20 \mathrm{Au} / 5 \mathrm{Ag} / 16 \mathrm{Fe} / \mathrm{GaAs}(001)$ is given mostly by the intrinsic bulk Gilbert damping. The FMR measurements at $24 \mathrm{GHz}$ resulted in $\alpha=4.5 \times 10^{-3}(\Delta H$ $=37 \mathrm{Oe}$ ). The FMR linewidth in $20 \mathrm{Au} / 300 \mathrm{Ag} / 16 \mathrm{Fe} / \mathrm{GaAs}$ was found to be increased to $\Delta H=45 \mathrm{Oe}$, showing the presence of spin-current relaxation to the Ag lattice. Clearly, the spin transport in the 300Ag spacer is not ballistic. Selfconsistent solution of Eqs. (7) and (8) allows one to estimate the spin-diffusion parameters. The following parameters were required to fit the measured FMR linewidths: $\tau_{\mathrm{sf}}=60$ $\times 10^{-14} \mathrm{~s}, \tau_{\mathrm{el}}=5 \times 10^{-14} \mathrm{~s}$, and the spin-diffusion length $\delta_{\text {sd }}=150 \mathrm{~nm}$. The spin mixing conductance $g_{\uparrow \downarrow}=1.2$ $\times 10^{15} \mathrm{~cm}^{-2}$ was taken from the $\mathrm{Fe} / \mathrm{Au} / \mathrm{Fe} / \mathrm{GaAs}(001)$ studies. ${ }^{8}$ The mixing conductance $g_{\uparrow \downarrow}$ is given by the number of electrons in NM spacer impinging the FM/NM interface. Since the Fermi surfaces in Au and Ag are very similar one can expect $g_{\uparrow \downarrow}$ in Ag and Au to be almost identical.

Using the same set of parameters as above one can try to explain the FMR linewidth, $\Delta H=64$ Oe at $f=24 \mathrm{GHz}$, for the $16 \mathrm{Fe}$ film in the $20 \mathrm{Au} / 12 \mathrm{Fe} / 300 \mathrm{Ag} / 16 \mathrm{Fe} / \mathrm{GaAs}$ structure when the FMR resonances were well separated [see Fig. $6(\mathrm{~b})]$. However in this sample surprisingly the $16 \mathrm{Fe}$ layer has a larger FMR linewidth, then the $12 \mathrm{Fe}$ film $(\Delta H=55 \mathrm{Oe})$. This seemingly contradicts the $1 / d$ dependence of the spinpumping contribution. However the total FMR linewidth is given by the sum of the bulk damping and interface spinpumping contributions. The spin-pumping contribution is a symmetric effect for the two Fe films and thus should remain the same. In order to explain the FMR linewidth of $12 \mathrm{Fe}$ one has to assume that the intrinsic Gilbert damping in $\mathrm{Au} /$ $12 \mathrm{Fe} / \mathrm{Ag}$ is smaller than that in $\mathrm{Ag} / 16 \mathrm{Fe} / \mathrm{GaAs}$. In $12 \mathrm{Fe}$ the required bulk Gilbert damping parameter $\alpha=2.5 \times 10^{-3}$. While for the $16 \mathrm{Fe}$ film the Gilbert damping parameter $\alpha$ $=4.5 \times 10^{-3}$. This result is not unexpected considering our recent studies of the intrinsic Gilbert damping in single $\mathrm{Fe}$ layer structures $[20 \mathrm{Au} / n \mathrm{Fe} / \mathrm{GaAs}(001)]$. In these structures the spin-pumping contribution was negligible compared to the intrinsic Gilbert damping. We found that the Gilbert damping in the Fe films has a significant $\frac{1}{d}$ contribution to the FMR linewidth (paper in preparation). In this case the observed thickness dependence is not caused by spin pumping but it originates due to additional extrinsic damping created by the $\mathrm{Fe} / \mathrm{GaAs}(001)$ interface chemistry. ${ }^{20}$ Obviously an equivalent $1 / d$ contribution to Gilbert damping in $\mathrm{Au} /$ $\mathrm{Fe} / \mathrm{Ag}$ structure is absent. It is interesting to note that a comparable value of the intrinsic Gilbert damping was found also in ultrathin Fe films grown on Ag single-crystal templates. ${ }^{21}$

Figure 6(b) shows the FMR signal for well-separated FMR peaks. Figure 6(c) shows the FMR signal at the cross- 
over of the FMR fields of the 16Fe and 12Fe layers [see the angular dependence in Fig. 6(a). At the crossover the spinpumping contribution to the magnetic damping is nearly canceled by the spin-sink contribution and the FMR linewidth drops by 17 and $14 \mathrm{Oe}$ for $16 \mathrm{Fe}$ and $12 \mathrm{Fe}$, respectively.

\section{MEASUREMENT OF THE SPIN-DIFFUSION LENGTH IN AU}

To obtain a full set of experimental data, which describes the spin-diffusion regime one has to use a NM spacer with the thickness comparable to the spin-diffusion length. Au has an appreciably larger atomic number (larger spin-orbit coupling) compared to $\mathrm{Ag}$ and consequently its $\delta_{\text {sd }}$ is expected to be smaller than in Ag. The values of $\delta_{\text {sd }}$ in Au obtained from the existing literature are found to be in the range from 10 to $170 \mathrm{~nm}$ at low temperatures. ${ }^{22}$ This wide spread in the data indicates that the measuring techniques and sample preparation affect the spin-diffusion data. Since the samples used here have an excellent single crystalline quality and the spin pumping is not accompanied with the net charge transport, it is desirable to investigate the propagation of spin currents using spin-pump/spin-sink effects and determine the spindiffusion parameters in Au spacers.

We carried out two types of experiments. (a) One is the FMR studies using $\mathrm{Au} / \mathrm{Fe} / \mathrm{GaAs}(001)$ structures. In this case one measures an increase in the interface Gilbert damping as a function of the Au overlayer thickness. Similar experiments were done by Mizukami et al. ${ }^{23}$ on polycrystalline $\mathrm{Cu} /$ Permalloy/Cu/Pt films. (b) The other is time, spatial, and depth-resolved Kerr effect (TRMOKE) studies using Au/Fe1/ $\mathrm{Au} / \mathrm{Fe} 2 / \mathrm{GaAs}(001)$. In this case one investigates the magnetization precession of the top $\mathrm{Fe} 1$ layer induced by the spin currents generated at the bottom layer $\mathrm{Fe} 2$ : $\mathrm{Fe} 2$ serves as a spin-current generator and the Fel layer serves as a spincurrent detection probe. The precessional motion of the magnetization in the Fe1 layer induced by pure spin currents was detected using TRMOKE. First results of these studies were presented in Ref. 14. Here we will present a more detailed account of this work including several new samples allowing one to carry out a more complete analysis of the spin diffusion in Au.

\section{A. FMR on $n \mathrm{Au} / 16 \mathrm{Fe} / \mathrm{GaAs}(001)$}

A series of samples with $n=20,80,150,200,250$, and 300 was prepared by MBE. The integers represent the number of atomic layers in the Au and Fe films. The FMR studies were carried out using standard microwave spectrometers at $10,24,36$, and $73 \mathrm{GHz}$ (see details in Ref. 19). The accumulated spin density in $\mathrm{Au}$, generated by spin pumping, is progressively attenuated with an increasing Au thickness by spin momentum scattering with phonons. This leads to a loss of the spin momentum in the Fe layer resulting in an increasing interface Gilbert damping. This increase eventually saturates when the Au layer thickness becomes larger than the spin-diffusion length $\delta_{\text {sd }}$ [see Fig. 7]. For $d_{\mathrm{NM}} \ll \delta_{\text {sd }}$ the FMR linewidth $\Delta H$ is given only by the intrinsic Gilbert damping of the $\mathrm{Fe}$ layer. One should point out that the increase in the

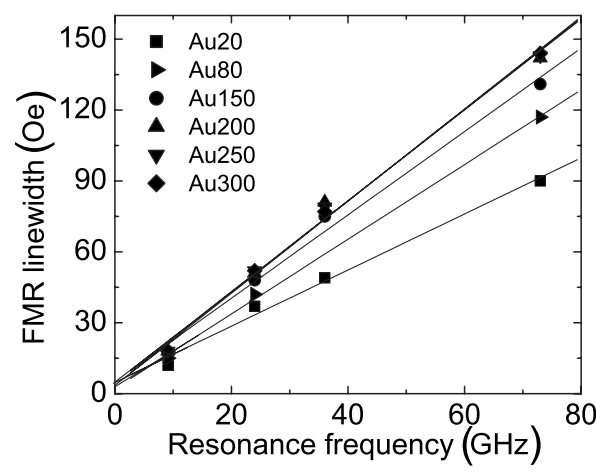

FIG. 7. FMR linewidth in $n \mathrm{Au} / 16 \mathrm{Fe} / \mathrm{GaAs}(001)$ vs microwave frequency as a function of the thickness of the Au capping layer, $n=20,80,150,200,250$, and 300 ML. The solid lines represent linear fits to frequency dependence of the FMR linewidth. The integers in the figure legend represent the number of atomic layers of the Au capping layer.

interface Gilbert damping first depends linearly on the $\mathrm{Au}$ layer thickness. Equations of motion (7) and (8) with boundary conditions (10) and (11) were used to extract the measured spin-pumping Gilbert damping coefficient $\alpha^{\mathrm{sp}}$ as a function of the Au layer thickness (see Fig. 8). Fitting resulted in the following parameters: $\alpha^{\text {int }}=3.5 \times 10^{-3}, g_{\uparrow \downarrow}$ $=1.2 \times 10^{15} \mathrm{~cm}^{-2}, \tau_{\mathrm{el}}=1.2 \times 10^{-14} \mathrm{~s}, \tau_{\mathrm{sf}}=15 \times 10^{-14} \mathrm{~s}$, and the Fermi velocity was assumed to be $v_{F}=1.4 \times 10^{8} \mathrm{~cm} / \mathrm{s}$. It is interesting to note that the relaxation times $\tau_{\mathrm{sf}}$ and $\tau_{\mathrm{el}}$ can be determined in a unique way. The reason for that is that the saturation value of $\alpha^{\mathrm{sp}}$ (measured for $300 \mathrm{ML}$ Au capping layer) is directly related to the ratio $\left(\tau_{\mathrm{sf}} / \tau_{\mathrm{el}}\right)^{0.5}$, while the approach to saturation (given by the spin-diffusion length $\left.\delta_{\mathrm{sd}}\right)$ is proportional to $\left(\tau_{\mathrm{el}} \tau_{\mathrm{sf}}\right)^{0.5 .5}$ The fitted parameters resulted in the spin-diffusion length $\delta_{\text {sd }}$ of $34 \mathrm{~nm}$ at room temperature.

Spin-diffusion constant $\tau_{\mathrm{sf}}$ is expected to be much larger than the electron momentum relaxation time $\tau_{\mathrm{el}}$. Indeed in our samples we determined $\tau_{\mathrm{sf}}$ to be a factor of 12 larger than that found for $\tau_{\mathrm{el}}$. The value of the momentum relaxation time is about a factor of 2 smaller than what is found in the bulk Au at RT. We will further discuss this result at the end of section TRMOKE studies.

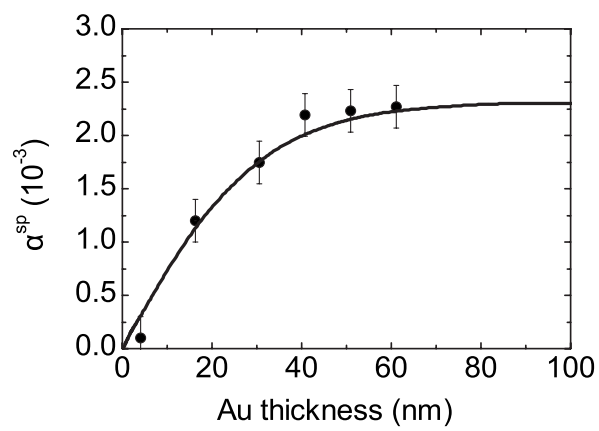

FIG. 8. Additional damping parameter in the $16 \mathrm{Fe}$ layer as a function of the thickness of the capping Au layer. The solid line shows a theoretical fit using the parameters $g_{\uparrow \downarrow}=1.2 \times 10^{15} \mathrm{~cm}^{-2}$, $\tau_{\mathrm{el}}=1.2 \times 10^{-14} \mathrm{~s}$, and $\tau_{\mathrm{sf}}=15 \times 10^{-14} \mathrm{~s}$, and the Fermi velocity was assumed to be $v_{F}=1.4 \times 10^{8} \mathrm{~cm} / \mathrm{s}$. 


\section{B. TRMOKE studies}

In trilayer structures one is able to observe not only the loss of spin momentum (due to spin pumping) but one can also directly study magnetic excitations by the spin-sink effect. Spin diffusion transports the accumulated spin density in $\mathrm{NM}$ to the $\mathrm{NM} / \mathrm{F} 2$ interface and providing an rf torque exciting the magnetic moment in Fe2. In the following experiments we studied the spin-sink effect in detail as a function of the Au spacer layer.

For the measurements, we employ temporal $(\sim 1 \mathrm{ps})$ and spatially $(\sim 300 \mathrm{~nm})$ resolved magneto-optical Kerr effect (TRMOKE) combined with continuous wave (cw) rf driving. The sample is excited by means of a cw rf field which is created by a comb generator from high harmonics of the 80 $\mathrm{MHz}$ Ti:sapphire laser repetition rate and is inherently phase locked to the laser probe pulses. A single rf from the comb generator is picked using a yttrium iron garnet filter and subsequently amplified. The magneto-optical Kerr signal is measured at a given delay time between the rf excitation and probing pulsed beam. In principle the amplitude of the observed signal at some arbitrary time is proportional to the transverse rf susceptibility $\chi$. In our studies we used the polar Kerr effect; this means the rf magnetization component perpendicular to the film surface was monitored as a function of time. The rf magnetization susceptibility $\chi=\chi^{\prime}-i \chi^{\prime \prime}$ consists of two parts: the $\chi^{\prime}$ is in phase with the rf driving field and $\chi^{\prime \prime}$ is $\pi / 2$ shifted with respect to the driving field. This means that the measured signal is given by

$$
\text { signal } \sim \chi^{\prime} \cos \phi+\chi^{\prime \prime} \sin \phi,
$$

where $\chi^{\prime}$ is the dispersive part, $\chi^{\prime \prime}$ is the absorptive part of the rf susceptibility, and $\phi$ is the phase angle $\phi$ between the driving rf field and the optical probing pulses. By selecting the correct microwave phase one is able to determine these two parts in separate measurements. If the probing light pulse is delayed such that its time of arrival coincides with the $\mathrm{rf}$ driving field maximum one observes dispersive part of $\chi^{\prime}$ and if the microwave phase is shifted from this position by a phase angle of $\pi / 2$ one observes $\chi^{\prime \prime}$. Thus by fixing the delay time for a given rf and sweeping the external dc magnetic field one can directly measure the in-phase and out-ofphase components of the transverse rf susceptibility. In principle this setup is similar to FMR but with one big difference, it allows one to measure a depth selective signal. In experiments with a pulsed magnetic excitation both magnetic layers (in double layer structure) are excited while using a cw microwave excitation allows one to selectively excite FMR dominantly in one layer of the double layer structure. For a given microwave frequency the particular FMR excitation is achieved by applying an appropriate dc magnetic field.

The position of the sample with respect to the microwave transmission line in our TRMOKE measurements is shown in Fig. 9(a). A small island of the measured sample is defined by optical lithography and dry etching. Subsequently, a 200nm-thick Au coplanar wave guide was prepared by optical lithography, thermal evaporation, and lift-off processes. The epitaxial layer structure is located in the gap of the coplanar waveguide between the signal conductor and the ground con-
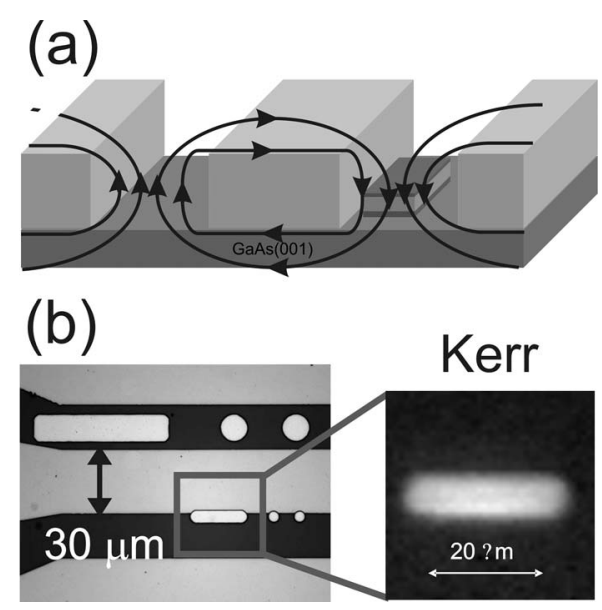

FIG. 9. (a) Schematic configuration of a transmission line and sample. The elliptical lines with the arrows show the rf driving field lines. (b) Optical micrograph of the patterned sample placed close to the coplanar waveguide. The magnified inset shows the magnetic Kerr signal response from the top $12 \mathrm{Fe}$ layer.

ductors [cf. Fig. 9(b)]. For this configuration the rf magnetic field is out of plane and in phase in both layers Fe1 and Fe2. The waveguide was oriented parallel to the [110] crystallographic direction of the Fe films and the magnetic dc field was applied parallel to the waveguide. An optical micrograph and the Kerr signal at FMR across a $25 \times 8 \mu \mathrm{m}^{2}$ epitaxial island is shown in Fig. 9(b). From the uniformity of the magnetic response it is evident that the dipolar fields are mostly created by edges of the sample and are negligible for ultrathin films studied in this paper.

TRMOKE measurements were carried out on $20 \mathrm{Au} / 12 \mathrm{Fe} / n \mathrm{Au} / 16 \mathrm{Fe} / \mathrm{GaAs}(001)$ magnetic double layers, where $n=150,200,250$, and 300 is the number of atomic layers. The difference in magnetic crystalline anisotropies for the top (12Fe) and bottom (16Fe) films was used to separate their FMR peaks. The top layer has a small uniaxial in-plane anisotropy, which is almost negligible compared to the other anisotropy fields. For in-plane FMR measurements with the dc field applied along the [110] crystallographic axis and neglecting small in-plane uniaxial anisotropy for the top layer the resonance conditions in the saturated state are given by

$$
\left(\frac{\omega}{\gamma}\right)^{2}=\left(H+4 \pi M_{\mathrm{eff}}+\frac{K_{1}}{M_{s}}\right) \times\left(H-\frac{2 K_{1}}{M_{s}} \pm \frac{2\left|K_{u}\right|}{M_{s}}\right),
$$

where (+) and (-) corresponds to the top $12 \mathrm{Fe}$ and bottom $16 \mathrm{Fe}$, respectively.

In order to study propagation of the spin current it is necessary to measure only the signal from the top $12 \mathrm{Fe}$ layer. This is not a trivial problem because the penetration depth of the $400 \mathrm{~nm}$ wavelength laser beam is on the order of $100 \mathrm{~nm}$. The residual signal contribution from the bottom layer even for a $200 \mathrm{ML} \mathrm{Au}$ spacer $(40 \mathrm{~nm})$ was about $10 \%$. A signal of this magnitude masks the spin-sink-driven signal. The residual Kerr signal from the bottom layer can be suppressed 
significantly below the level of the spin-current-induced signal by employing the depth selectivity of the Kerr effect. One can adjust the phase of the Kerr polarization with respect to the reference light beam reflected from the film surface. It is important to realize that the polarization of the reference and Kerr signals are perpendicular to each other. In this case a rotatable compensator (e.g., 1/4 wave plate) positioned between the sample and the analyzer allows one to adjust the phase between these two signals. Hamrle et al. ${ }^{24}$ showed that in multilayer films the Fe film under a thick $\mathrm{Au}$ cap layer generates a Kerr signal with a phase distinctly different from the Fe layer which is close to the sample surface. This phase difference originates in different optical path lengths of the corresponding Kerr signals. A careful adjustment of the $1 / 4$ wave plate allowed one to set the phase of the Kerr signal from the bottom layer with the phase which was $\frac{\pi}{2}$ shifted with respect to the reference beam polarization. In this case the bottom layer signal becomes negligible because it is in quadrature with the reference signal. Using this intrinsic phase shift in the Kerr polarizations one is able to select the signal only from the top layer. This technique was first demonstrated by Hubert and $\mathrm{Shafer}^{25}$ for $\mathrm{Fe} / \mathrm{Cr} / \mathrm{Fe}$ multilayers.

In the field sweep measurement one can observe apart from the main FMR signal due to the top 12Fe layer (at the external field $\simeq 1 \mathrm{kOe}$ ) the magnetization precession induced in the top $12 \mathrm{Fe}$ layer by spin currents generated by the bottom $16 \mathrm{Fe}$ layer (at the external field $\simeq 0.5 \mathrm{kOe}$ ). The spin-sink-induced signal occurs at the FMR position of the bottom $16 \mathrm{Fe}$ layer because the accumulated magnetic moment in the Au spacer follows the FMR response of the bottom $16 \mathrm{Fe}$ layer. The spin-current-driven signal is proportional to $\frac{d \tilde{m}_{j}}{d t}$. This means that the $\chi^{\prime}$ susceptibility corresponding to the top $12 \mathrm{Fe}$ layer TRMOKE signal is accompanied by the spin-sink signal component $\sim \chi^{\prime \prime}$ of the bottom $16 \mathrm{Fe}$ layer and vice versa. It follows that the direct TRMOKE signal from the bottom $16 \mathrm{Fe}$ layer, if observable, is phase shifted by $\pi / 2$ from the spin-current-induced signal. This feature was used to distinguish between the signals generated by the direct observation of the Kerr effect from the bottom layer and the spin-sink signal. In fact, the 1/4 wave plate was adjusted to such a position that the direct Kerr signal from the bottom 16Fe layer was suppressed. Only the spin-sink signal, which was $\frac{\pi}{2}$ shifted with respect to the main TRMOKE signal, was observed ${ }^{14}$ (see Fig. 10).

The TRMOKE experiments were carried out at $10.3 \mathrm{GHz}$. The resonance fields for both magnetic films were calculated using Eq. (14). They were separated by $\approx 450$ Oe. The results for the $\chi^{\prime}$ and $\chi^{\prime \prime}$ signals for the $20 \mathrm{Au} / 12 \mathrm{Fe} / 200 \mathrm{Au} /$ $16 \mathrm{Fe} / \mathrm{GaAs}$ sample are shown in Fig. 10. Apart from the main FMR signal, one can clearly observe the spin-sinkinduced signal in the tail of the main FMR signal. The position of the spin-sink-induced signal perfectly agrees with the FMR position of the bottom $16 \mathrm{Fe}$ layer, calculated from Eq. (14). The top $12 \mathrm{Fe}$ layer was driven by the spin current generated by the bottom $16 \mathrm{Fe}$ layer [see Eq. (7)], which is proportional to the time derivative of the rf magnetization of the $16 \mathrm{Fe}$ layer. Therefore this driving was phase shifted by $\pi / 2$ with respect to the rf magnetization of the top $12 \mathrm{Fe}$ layer.
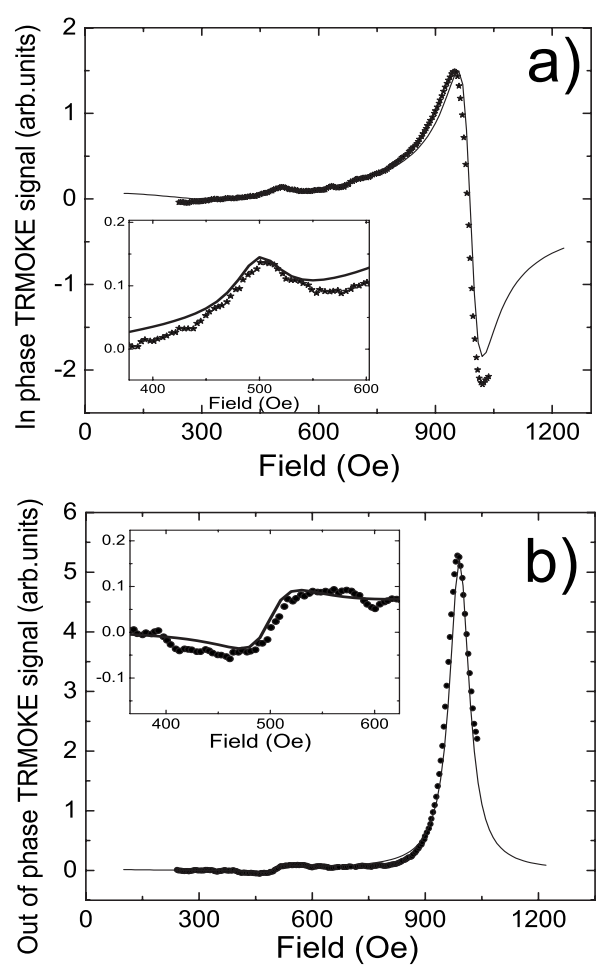

FIG. 10. TRMOKE field scan at $10.3 \mathrm{GHz}$ for the $20 \mathrm{Au} / 12 \mathrm{Fe} /$ $200 \mathrm{Au} / 16 \mathrm{Fe} / \mathrm{GaAs}$ sample. The magnetic field was oriented along the easy axis $([110])$ of the bottom $16 \mathrm{Fe}$ layer. The $1 / 4$ wave plate was adjusted to suppress the direct signal from the bottom $16 \mathrm{Fe}$ layer below our detection limit. The magnetocrystalline anisotropies for both magnetic films were determined using FMR measurements and are as follows: $16 \mathrm{Fe}$ grown on $\mathrm{GaAs}(001)$. In-plane uniaxial anisotropy $K_{u}=-4.45 \times 10^{5} \mathrm{erg} / \mathrm{cm}^{3}$ with the hard magnetic axis along the $[1 \overline{1} 0]_{\mathrm{GaAs}}$, the in-plane fourfold anisotropy $K_{1}=2.67$ $\times 10^{5} \mathrm{erg} / \mathrm{cm}^{3}$, and effective demagnetizing field $4 \pi M_{\mathrm{eff}}$ $=16.94 \mathrm{kG}$. $12 \mathrm{Fe}$ film grown on the $\mathrm{Au}(001)$ layer: $K_{1}=2.49$ $\times 10^{5} \mathrm{erg} / \mathrm{cm}^{3}, K_{u}=8.63 \times 10^{3} \mathrm{erg} / \mathrm{cm}^{3}$ with the easy magnetic axis along $[1 \overline{1} 0]_{\mathrm{GaAs}}$ and $4 \pi M_{\mathrm{eff}}=16.41 \mathrm{kG}$. (a) shows $\chi^{\prime}$ which is in phase with the rf driving field. (b) shows $\chi^{\prime \prime}$, which is phase shifted by $\frac{\pi}{2}$ with respect to the rf driving field. The insets show the signal due to the spin-sink mechanism at a magnified scale. The solid lines show the results of computer simulation using Eq. (8) with boundary conditions (10) and (12).

Consequently, the spin-sink-induced precession resulted in a typical "dispersion wiggle" for the imaginary part of the $12 \mathrm{Fe}$ susceptibility $\chi^{\prime}$ and a "dip" for the real part of the $12 \mathrm{Fe}$ susceptibility. The solid lines show computer fits of the data using Eqs. (7) and (8) with boundary conditions (10) and (12) using the following parameters: $\tau_{\mathrm{el}}=1.2 \times 10^{-14} \mathrm{~s}$ and $\tau_{\mathrm{sf}}=15 \times 10^{-14} \mathrm{~s}$. These fitting parameters are very close to those obtained from the FMR measurements on the Au/ $\mathrm{Fe} / \mathrm{GaAs}(001)$ samples. In the limit of ballistic spin transport one would expect the magnitude of the spin-sink-driven signal to be approximately three times larger than that observed in our measurements.

The spin-current-induced dynamics was studied as a function of the Au spacer layer thickness (see Fig. 11). The intensity of the spin-current-induced TRMOKE signal was normalized to the intensity of $\chi^{\prime \prime}$ of the main TRMOKE signal 


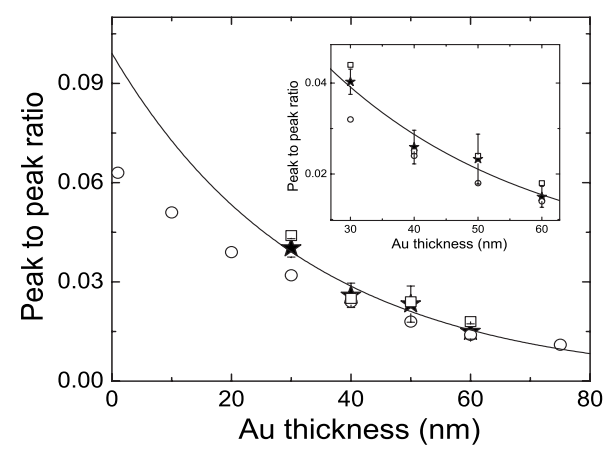

FIG. 11. The $\star$ points show the experimental peak-to-peak ratio of the signal driven by the spin-sink mechanism to the main FMR resonance as a function of the Au spacer layer. The solid line shows a simple exponential decay fit to the experimental data. The $\bigcirc$ points show the result of simulations, which assume perfect alignment of the magnetic moment in the top $12 \mathrm{Fe}$ film with respect to the external field. The $\square$ points show the result of simulations which take into account the separation in the magnetic field between the main FMR peak and the signal driven by the spin-sink mechanism. These simulations include dragging of the magnetic moment in the top $12 \mathrm{Fe}$ layer behind the applied dc field.

due to the top $12 \mathrm{Fe}$ layer to account for different optical reflectivities for samples with different spacer layers. In Fig. 11 we plot the ratio of the peak-to-peak intensity of the spincurrent-induced signal to the peak to base intensity of the main signal from the top 12Fe layer [see Fig. 10(b)]. The data points for different thicknesses of the $\mathrm{Au}$ spacer layer are shown as $\star$. The strength of the spin-current-induced signal decreased with an increasing spacer thickness. A simple exponential fit to the data provides a good fit; however, it leads to an unrealistically strong contribution in the limit of small thicknesses corresponding to ballistic spin transport. The spin-diffusion theory using a perfect alignment between the magnetic moments and the applied dc field did not result in a satisfying fit (see the points $\circ$ in Fig. 11). A better fit requires to include dragging of the magnetic moment in the top $12 \mathrm{Fe}$ layer behind the external magnetic field. The bottom layer was oriented with the field along the in-plane easy axis. The applied field in the top layer was applied along the hard axis and consequently due to an imperfect alignment its magnetic moment was dragged behind the applied field and was not collinear with the magnetic moment of the $16 \mathrm{Fe}$ layer. This layer gets fully aligned with the magnetic field only for perfect orientation of the field along the hard axes $(\langle 110\rangle)$ and $H>2 K_{1} / M_{s}$. The bottom layer was very little affected because it was oriented along its easy magnetic axis. A misalignment of the magnetic moment with respect to the direction of the dc field within $10^{\circ}$ already resulted in a significant change in the relative amplitudes of the main (FMR) and satellite (spin-sink-driven) signals. The misalignment angle of the field and crystallographic axes was identified by the shift of the resonant field of the top Fe layer from its expected value for the hard magnetic axis ([110]). The full fit using misalignment of the magnetic moment in the top 12Fe layer (see the $\square$ points in Fig. 11) resulted in a good fit to the data using the following set of spin-diffusion parameters: the spin-flip relaxation time $\left(\tau_{\mathrm{sf}}=15 \times 10^{-14} \mathrm{~s}\right)$, electron relaxation time $\left(\tau_{\mathrm{el}}=1.2\right.$ $\left.\times 10^{-14} \mathrm{~s}\right)$, and the spin-diffusion length $\left(\delta_{\mathrm{sd}}\right.$ of $\left.34 \mathrm{~nm}\right)$ at room temperature.

The electron momentum relaxation time $\tau_{\mathrm{el}}$ obtained from the FMR and TRMOKE data can be compared to the data obtained from the sheet resistance measurements in quantitative giant magnetoresistance (GMR) studies using Fe/Au/ $\mathrm{Fe} / \mathrm{GaAs}(001)$ multilayers. ${ }^{26}$ The value for the electron relaxation time $\tau_{\mathrm{el}}$ can be also obtained independently from the expression for the electrical conductivity in $\mathrm{Au} \sigma_{\mathrm{Au}}$,

$$
\sigma_{\mathrm{Au}}=\frac{n e^{2} \tau_{\mathrm{el}}}{m},
$$

where $n=5.9 \times 10^{28} \mathrm{~m}^{-3}$ is the charge-carrier density per unit volume, $e$ is the electron charge, and $m$ is the mass of the electron. Using $\sigma_{\mathrm{Au}}=0.44 \times 10^{8}(\Omega \mathrm{m})^{-1}$ obtained from the literature ${ }^{27}$ results in the momentum relaxation $\tau_{\mathrm{el}}=2.3$ $\times 10^{-14}$, which is almost two times longer than $\tau_{\mathrm{el}}=1.2$ $\times 10^{-14}$ s obtained in our experiments. Monchesky et al. $^{26}$ showed that in the $\mathrm{Au}(001)$ films grown on $\mathrm{GaAs} / \mathrm{Fe}(001)$ templates the sheet conductivity decreases with decreasing thickness of Au due to interface scattering. In the range of 20-30 nm the average value of the conductivity $\sigma_{\mathrm{Au}}=0.3$ $\times 10^{8}(\Omega \mathrm{m})^{-1}$. Using Eq. (15) and the thickness adjusted $\sigma_{\mathrm{Au}}$ we get $\tau_{\mathrm{el}}=1.6 \times 10^{-14}$, which is in good agreement with the result obtained from our dynamic magnetic measurements.

Kurt et $a .^{28}$ studied the spin-diffusion length by using CPP (current perpendicular to plane) GMR measurements with polycrystalline $\mathrm{Au} / \mathrm{Cu}$ spacers. They obtained $\delta_{\text {sd }}$ $=35 \mathrm{~nm}$ at $4.2 \mathrm{~K}$. Extrapolation of these data to room temperature should result in a decreased value. The smaller spindiffusion length in their experiment compared to the present results may be explained by additional scattering on grain boundaries of the polycrystalline $\mathrm{Au} / \mathrm{Cu}$ spacer. Our results are in good agreement with Au spin-diffusion measurements performed in lateral spin valves by $\mathrm{Ji}$ et al. ${ }^{29}$ These authors report $\delta_{\mathrm{sd}}=64 \mathrm{~nm}$ at $10 \mathrm{~K}$. From other experiments one can conclude that the spin-diffusion length at low temperatures is about a factor of 2 larger than at room temperature. ${ }^{30}$ If one applies this rule to the Au measurements one obtains $\delta_{\text {sd }}$ $=32 \mathrm{~nm}$ at room temperature, which is very close to our results.

\section{CONCLUSIONS}

The magnetic damping in double magnetic layer structures $(\mathrm{Fe} / \mathrm{NM} / \mathrm{Fe} / \mathrm{GaAs})$ consists of two contributions: (a) Gilbert damping due to spin-orbit coupling and (b) nonlocal damping due to spin-pump mechanism. We found that the Gilbert damping in the Fe films grown directly on the $4 \times 6$ reconstructed GaAs substrate has a significant $1 / d$ contribution. In this case the observed $1 / d$ dependence is not due to spin pumping but is caused by the interface electron band structure of $\mathrm{Au} / \mathrm{Fe} / \mathrm{GaAs}(001)$ and will be reported in an upcoming paper. The spin-pump/spin-sink mechanism leads to an additional source of interface damping in magnetic structures. Spin currents created at the F/NM interface lead to an accumulated spin density inside the nonmagnetic spacer and 
can result in nonlocal damping. The accumulated spin momentum density propagation was studied in magnetic single and double layers. We conclude that the propagation of the accumulated magnetic-momentum density can be described by a spin-diffusion equation. Simulations based on the spindiffusion theory are in good agreement with the experimental data. Computer fits of the experimental data revealed a number of important parameters for the crystalline $\mathrm{Au}(001)$ spacers at room temperature: the spin-flip relaxation time $\tau_{\text {sf }}$ $=15 \times 10^{-14} \mathrm{~s}$, the electron relaxation time $\tau_{\mathrm{el}}=1.2$ $\times 10^{-14} \mathrm{~s}$, and a spin-diffusion length $\delta_{\text {sd }}$ of $34 \mathrm{~nm}$. The direct detection of spin currents was carried out by using depth resolved TRMOKE. The results are in quantitative agreement with self-consistent calculations based on the spin-pump/spin-sink theory and the spin-diffusion equation. We have shown that the depth resolved TRMOKE technique is a unique tool allowing one to study the spin diffusion in normal metals using magnetic double layer structures.

\section{ACKNOWLEDGMENTS}

The authors would like to thank the National Science and Engineering Research Council of Canada (NSERC) and Canadian Institute for Advanced Research (CIFAR) for generous grants which supported this work. Financial support by the DFG priority program through Grant No. SPP1133 and Sonderforschungsbereich through Grant No. SFB689 is gratefully acknowledged.
${ }^{1}$ B. Heinrich and J. F. Cochran, Adv. Phys. 42, 523 (1993).

${ }^{2}$ B. Heinrich, in Magnetic Ultrathin Film Structures, edited by J. A. C. Bland and B. Heinrich (Springer, New York, 2004), Vol. III.

${ }^{3}$ Y. Tserkovnyak, A. Brataas, and G. E. W. Bauer, Phys. Rev. Lett. 88, 117601 (2002).

${ }^{4}$ B. Heinrich, Y. Tserkovnyak, G. Woltersdorf, A. Brataas, R. Urban, and G. E. W. Bauer, Phys. Rev. Lett. 90, 187601 (2003).

${ }^{5}$ Y. Tserkovnyak, A. Brataas, G. Bauer, and B. Halperin, Rev. Mod. Phys. 77, 1375 (2005).

${ }^{6}$ M. D. Stiles and A. Zangwill, Phys. Rev. B 66, 014407 (2002).

${ }^{7}$ B. Heinrich, in Ultrathin Magnetic Structures, edited by B. Heinrich and J. F. C. Bland (Springer, New York, 2003), Vol. III.

${ }^{8}$ R. Urban, G. Woltersdorf, and B. Heinrich, Phys. Rev. Lett. 87, 217204 (2001).

${ }^{9}$ R. Urban, B. Heinrich, and G. Woltersdorf, J. Appl. Phys. 93, 8280 (2003).

${ }^{10}$ S. Mizukami, Y. Ando, and T. Miyazaki, J. Magn. Magn. Mater. 239, 42 (2002).

${ }^{11}$ M. V. Costache, M. Sladkov, S. M. Watts, C. H. van der Wal, and B. J. van Wees, Phys. Rev. Lett. 97, 216603 (2006).

${ }^{12}$ A. Brataas, Y. Tserkovnyak, G. E. W. Bauer, and B. I. Halperin, Phys. Rev. B 66, 060404(R) (2002).

${ }^{13}$ E. Šimánek and B. Heinrich, Phys. Rev. B 67, 144418 (2003).

${ }^{14}$ G. Woltersdorf, O. Mosendz, B. Heinrich, and C. H. Back, Phys. Rev. Lett. 99, 246603 (2007).

${ }^{15}$ B. Kardasz, O. Mosendz, B. Heinrich, Z. Liu, and M. Freeman, J. Appl. Phys. 103, 07C509 (2008).

${ }^{16}$ R. J. Elliott, Phys. Rev. 96, 266 (1954).

${ }^{17}$ Y. Tserkovnyak, A. Brataas, and G. E. W. Bauer, Phys. Rev. B
66, 224403 (2002).

${ }^{18}$ G. Woltersdorf, B. Heinrich, J. Woltersdorf, and R. Scholz, J. Appl. Phys. 95, 7007 (2004).

${ }^{19}$ B. Heinrich and J. A. C. Bland, Ultrathin Magnetic Structures (Springer-Verlag, Berlin, 1994), Vol. II, pp. 195-222.

${ }^{20}$ B. Kardasz, J. Zukrowski, O. Mosendz, M. Przbylski, B. Heinrich, and J. Kirschner, J. Appl. Phys. 101, 09D110 (2007).

${ }^{21}$ B. Heinrich, K. B. Urquhart, A. S. Arrott, J. F. Cochran, K. Myrtle, and S. T. Purcell, Phys. Rev. Lett. 59, 1756 (1987).

${ }^{22}$ J. Bass and W. Pratt, J. Phys.: Condens. Matter 19, 183201 (2007).

${ }^{23}$ S. Mizukami, Y. Ando, and T. Miyazaki, Phys. Rev. B 66, 104413 (2002).

${ }^{24}$ J. Hamrle, J. Ferre, M. Nyvlt, and S. Visnovsky, Phys. Rev. B 66, 224423 (2002).

${ }^{25}$ A. Hubert and R. Shafer, Magnetic Domains: The Analysis of Magnetic Microstrucutres (Springer, New York, 1998).

${ }^{26}$ T. L. Monchesky, A. Enders, R. Urban, K. Myrtle, B. Heinrich, X. G. Zhang, W. H. Butler, and J. Kirschner, Phys. Rev. B 71, 214440 (2005).

${ }^{27}$ CRC Handbook of Chemistry and Physics, edited by R. C. Weast, M. J. Astle, and W. H. Beyer (CRC, Boca Raton, FL, 1985).

${ }^{28}$ H. Kurt, W.-C. Chiang, C. RItz, K. Eid, W. Pratt, and J. Bass, J. Appl. Phys. 93, 7918 (2003).

${ }^{29}$ Y. Ji, A. Hoffmann, J. Jiang, and S. Bader, Appl. Phys. Lett. 85, 6218 (2004).

${ }^{30}$ Y. Ji, A. Hoffmann, J. Jiang, J. Pearson, and S. Bader, J. Phys. D 40, 1280 (2007). 